\title{
Effects of Docosahexaenoic Acid on the Endothelial Function in Patients with Coronary Artery Disease
}

\author{
Shusuke Yagi ${ }^{1}$, Ken-ichi Aihara ${ }^{2}$, Daiju Fukuda ${ }^{3}$, Akira Takashima $^{1}$, Tomoya Hara ${ }^{1}$, Junko Hotchi ${ }^{1}$, \\ Takayuki Ise $^{1}$, Koji Yamaguchi ${ }^{1}$, Takeshi Tobiume ${ }^{1}$, Takashi Iwase ${ }^{1}$, Hirotsugu Yamada ${ }^{1}$, Takeshi Soeki ${ }^{1}$, \\ Tetsuzo Wakatsuki ${ }^{1}$, Michio Shimabukuro ${ }^{3}$, Masashi Akaike ${ }^{4}$ and Masataka Sata ${ }^{1}$
}

\begin{abstract}
${ }^{1}$ Department of Cardiovascular Medicine, the University of Tokushima Graduate School of Health Biosciences, Tokushima, Japan
${ }^{2}$ Department of Medicine and Bioregulatory Sciences, the University of Tokushima Graduate School of Health Biosciences, Tokushima, Japan

${ }^{3}$ Department of Cardio-Diabetes Medicine, the University of Tokushima Graduate School of Health Biosciences, Tokushima, Japan ${ }^{4}$ Department of Medical Education, the University of Tokushima Graduate School of Health Biosciences, Tokushima, Japan
\end{abstract}

\begin{abstract}
Aim: The consumption of $\mathbf{n}-3$ polyunsaturated fatty acids (PUFA), including docosahexaenoic acid (DHA), reduces the incidence of cardiovascular events, and reduced serum levels of n-3 PUFA may be associated with an increased risk of cardiovascular events. However, controversy remains regarding which components of PUFA are associated with the endothelial function in patients with coronary artery disease (CAD). We therefore examined the associations between the n-3 and n-6 PUFA levels and CAD.

Methods: We retrospectively reviewed 160 consecutive Japanese patients with CAD whose endothelial function was measured according to the percent change in flow-mediated dilation (FMD) and the serum levels of n-3 PUFA, including eicosapentaenoic acid (EPA) and DHA, and n-6 PUFA, including arachidonic acid (AA) and dihomo-gamma-linolenic acid (DHLA).

Results: A single regression analysis showed no relationships between the FMD and the serum levels of PUFA, including EPA, DHA, AA and DHLA. In contrast, a multiple regression analysis showed that the DHA level was a positive $(P<0.01)$ and age was a negative $(P<0.001)$ contributor to an increased FMD; however, sex, body mass index, systolic and diastolic blood pressure, current/past smoking and the levels of HbA1c, triglycerides, low-density lipoprotein cholesterol, high-density lipoprotein cholesterol, EPA, AA and DHLA did not significantly affect the outcome.

Conclusions: The serum level of DHA is associated with the endothelial function evaluated according to the FMD in patients with CAD, thus suggesting that a low serum level of DHA may be a predictive biomarker for endothelial dysfunction.
\end{abstract}

\section{See editorial vol. 22: 440-441}

J Atheroscler Thromb, 2015; 22: 447-454.

Key words: Flow-mediated dilation, n-3 polyunsaturated fatty acids, Docosahexaenoic acid

\section{Introduction}

Despite advances in reducing the low-density

Address for correspondence: Shusuke Yagi, Department of Cardiovascular Medicine, the University of Tokushima Graduate School of Health Biosciences, 3-18-15 Kuramotocho, Tokushima-city, Tokushima 770-8503, Japan

E-mail: syagi@tokushima-u.ac.jp

Received: July 4, 2014

Accepted for publication: September 15, 2014 lipoprotein cholesterol (LDL-C) levels to below target ranges with lipid-lowering agents, including HMGCoA reductase inhibitors, the incidence of cardiovascular events remains high. Therefore, other therapies to reduce cardiovascular events should be identified ${ }^{1)}$.

Vascular endothelial dysfunction is recognized to be a critical factor in the development of coronary artery disease $(\mathrm{CAD})^{2}$, and evaluating the endothelial function is therefore important for determining the risk of CAD. Recently, the flow-mediated dilation 
(FMD) has become widely used as a noninvasive method to assess the brachial arterial endothelial function by measuring the diameter on ultrasound before and after a period of induced ischemia of the forearm. The degree of dilation is mediated by endothelial nitric oxide (NO) release in response to increased shear stress ${ }^{3,4)}$. As the FMD is thought to reflect the ability of the endothelium to release NO, which regulates vascular tone, this parameter may be a useful surrogate marker for cardiovascular events ${ }^{5,6}$.

It has been reported that reduced serum levels of n-3 polyunsaturated fatty acids (PUFA), including eicosapentaenoic acid (EPA) and docosahexaenoic acid (DHA), are associated with an increased incidence of cardiovascular events and mortality ${ }^{7,8)}$, while supplementation of n-3 PUFA improves the endothelial function ${ }^{9}$, 10). Therefore, the cardiovascular event risklowering effects of n-3 PUFA may be mediated partly via endothelial-dependent mechanisms, and reduced serum levels of n-3 PUFA may be associated with an increased risk of cardiovascular events in patients with CAD. In addition, n-6 PUFA, including arachidonic acid (AA) and dihomo-gamma-linolenic acid (DHLA), are known to be proinflammatory fatty acids, and their effects on the cardiovascular system remain controversial ${ }^{11)}$.

Hence, to elucidate which components of PUFA are associated with the endothelial function in patients with $\mathrm{CAD}$, we evaluated the association between each PUFA component, including EPA, DHA, AA and DHLA, and the endothelial function in patients with CAD.

\section{Methods}

We retrospectively reviewed 160 consecutive Japanese patients with CAD who had undergone measurements of the FMD and serum levels of PUFA. The patients were recruited from the Department of Cardiology at Tokushima University Hospital between October 2012 and May 2013.

Patients with CAD were defined as having a history of myocardial infarction, angiographic evidence of $\geq 50 \%$ stenosis in $\geq 1$ coronary artery, evidence of exercise-induced ischemia or a history of coronary revascularization. The exclusion criteria were as follows: the use of fish oil supplements or n-3 fatty acidcontaining drugs or a history of myocardial infarction within the past month. In addition, patients with symptomatic active malignant diseases, renal failure (serum creatinine level $>4.0 \mathrm{mg} / \mathrm{dL}$ ) or liver dysfunction (aspartate aminotransferase level $>100 \mathrm{IU} / \mathrm{L}$, alanine aminotransferase level $>100 \mathrm{IU} / \mathrm{L}$ ) were excluded.
Blood pressure was measured in the sitting position according to previously described guidelines ${ }^{12)}$. Hypertensive patients were defined as those with a systolic blood pressure of $\geq 140 \mathrm{~mm} \mathrm{Hg}$ and/or diastolic blood pressure of $\geq 90 \mathrm{~mm} \mathrm{Hg}$ and/or currently receiving antihypertensive medications. Dyslipidemic patients were defined as those with an LDL-C level of $\geq 140 \mathrm{mg} / \mathrm{dL}$, triglyceride level of $\geq 150 \mathrm{mg} / \mathrm{dL}$, highdensity lipoprotein cholesterol (HDL-C) level of $<40$ $\mathrm{mg} / \mathrm{dL}$ or currently receiving lipid-lowering medications. Diabetic patients were defined as individuals receiving insulin or oral hypoglycemic agents or those with an HbA1c level of $\geq 6.5 \%$, fasting plasma glucose level of $126 \mathrm{mg} / \mathrm{dL}$ or casual plasma glucose level of $\geq 200 \mathrm{mg} / \mathrm{dL}$. The smoking status (current or past smoker) was evaluated using a questionnaire. The body mass index was calculated as an index of obesity.

The FMD of the brachial artery was measured to assess endothelium-dependent vascular reactivity using a high-resolution ultrasound system coupled to a computer-assisted analysis software program (UNEXEF18G, UNEX Co, Nagoya, Japan). The measurements were obtained by trained vascular ultrasonographers according to the recommendations of the International Brachial Artery Reactivity Task Force Guidelines for the ultrasound assessment of endothelialdependent FMD vasodilatation of the brachial artery, as described elsewhere ${ }^{13,14)}$.

The levels of four bioactive serum fatty acids, including EPA, DHA, AA and DHLA, were measured using a gas - liquid chromatograph (GC-2010; Shimadzu, Kyoto, Japan) equipped with a capillary column (TC-70; GL Sciences, Tokyo, Japan) at a commercially available laboratory (SRL, Tokyo, Japan). Because AA, an n-6 PUFA, is a proatherosclerotic fatty acid, a reduced EPA/AA ratio is widely used as a biomarker for the development of CAD ${ }^{15)}$. The EPA/ $\mathrm{AA}$ and DHA/AA ratios were therefore calculated.

In addition, other biochemical parameters, including the levels of LDL-C, triglycerides, HDL-C, random plasma glucose, $\mathrm{HbA1c}$ and serum creatinine, were measured.

Written informed consent was not required due to the retrospective nature of the investigation. This study protocol was approved by the Tokushima University Hospital Ethics Committee in accordance with the Declaration of Helsinki.

\section{Statistical Analysis}

Continuous variables were averaged and expressed as the mean \pm standard deviation $(\mathrm{SD})$ or as a percentage for categorical parameters. A single 
Table 1. Clinical characteristics of the patients

\begin{tabular}{|c|c|}
\hline \multicolumn{2}{|l|}{ Variable } \\
\hline Number of patients & 160 \\
\hline Male & $123(76.9 \%)$ \\
\hline Age, (y) & $70.3 \pm 9.4$ \\
\hline Body mass index, $\left(\mathrm{kg} / \mathrm{m}^{2}\right)$ & $23.1 \pm 4.7$ \\
\hline Systolic blood pressure, $(\mathrm{mmHg})$ & $131.7 \pm 18.4$ \\
\hline Diastolic blood pressure, $(\mathrm{mmHg})$ & $74.5 \pm 10.1$ \\
\hline Low-density lipoprotein cholesterol, (mg/dL) & $98.9 \pm 24.5$ \\
\hline Triglycerides, (mg/dL) & $131.7 \pm 59.1$ \\
\hline High-density lipoprotein cholesterol, (mg/dL) & $54.1 \pm 16.5$ \\
\hline HbA1c, (\%) & $6.5 \pm 0.90$ \\
\hline Random plasma blood glucose, (mg/dL) & $122.1 \pm 40.6$ \\
\hline Serum creatinine, $(\mathrm{mg} / \mathrm{dL})$ & $0.87 \pm 0.30$ \\
\hline \multicolumn{2}{|l|}{ Fatty acid concentrations } \\
\hline DHA, $(\mu \mathrm{g} / \mathrm{mL})$ & $128.5 \pm 43.2$ \\
\hline $\mathrm{EPA},(\mu \mathrm{g} / \mathrm{mL})$ & $57.5 \pm 29.7$ \\
\hline $\mathrm{AA},(\mu \mathrm{g} / \mathrm{mL})$ & $167.7 \pm 44.3$ \\
\hline DHLA, $(\mu \mathrm{g} / \mathrm{mL})$ & $34.7 \pm 12.0$ \\
\hline EPA/AA & $0.37 \pm 0.22$ \\
\hline DHA/AA & $0.81 \pm 0.29$ \\
\hline FMD, (\%) & $4.1 \pm 2.2$ \\
\hline \multicolumn{2}{|l|}{ Comorbidities } \\
\hline Dyslipidemia & $130(81.3 \%)$ \\
\hline Hypertension & $132(82.5 \%)$ \\
\hline Diabetes mellitus & $73(45.6 \%)$ \\
\hline Current/past smoker & $102(63.8 \%)$ \\
\hline \multicolumn{2}{|l|}{ Drugs taken } \\
\hline HMG-CoA reductase inhibitor & $134(83.8 \%)$ \\
\hline Ezetimibe & $14(8.8 \%)$ \\
\hline Fibrate & $1(0.6 \%)$ \\
\hline ACE-I/ARB & $112(70.0 \%)$ \\
\hline$\beta$-blocker & $58(36.3 \%)$ \\
\hline Calcium channel blocker & $60(37.5 \%)$ \\
\hline
\end{tabular}

The values are presented as the mean $\pm \mathrm{SD}$ or $n(\%)$ as appropriate. HbA1c, hemoglobin A1c; DHA, docosahexaenoic acid; EPA, eicosapentaenoic acid; AA, arachidonic acid; DHLA, dihomo-gamma-linolenic acid; FMD, flow-mediated dilation; ACE-I, angiotensin-converting enzyme inhibitor; $\mathrm{ARB}$, angiotensin II receptor blocker

regression analysis was used to assess the correlations between the FMD and the CAD risk factors. A multiple regression analysis was used to assess the degree of association between the FMD and the CAD risk factors. The Kolmogorov-Smirnov test was performed to evaluate whether the parameters were normally distributed. Non-normally distributed parameters, including the FMD, systolic blood pressure, triglycerides, HDL-C, HbAc1, EPA, EPA/AA, DHA and DHA/AA, were natural log transformed and analyzed. All statistical analyses were performed using the JMP 10 software program. Statistical significance was
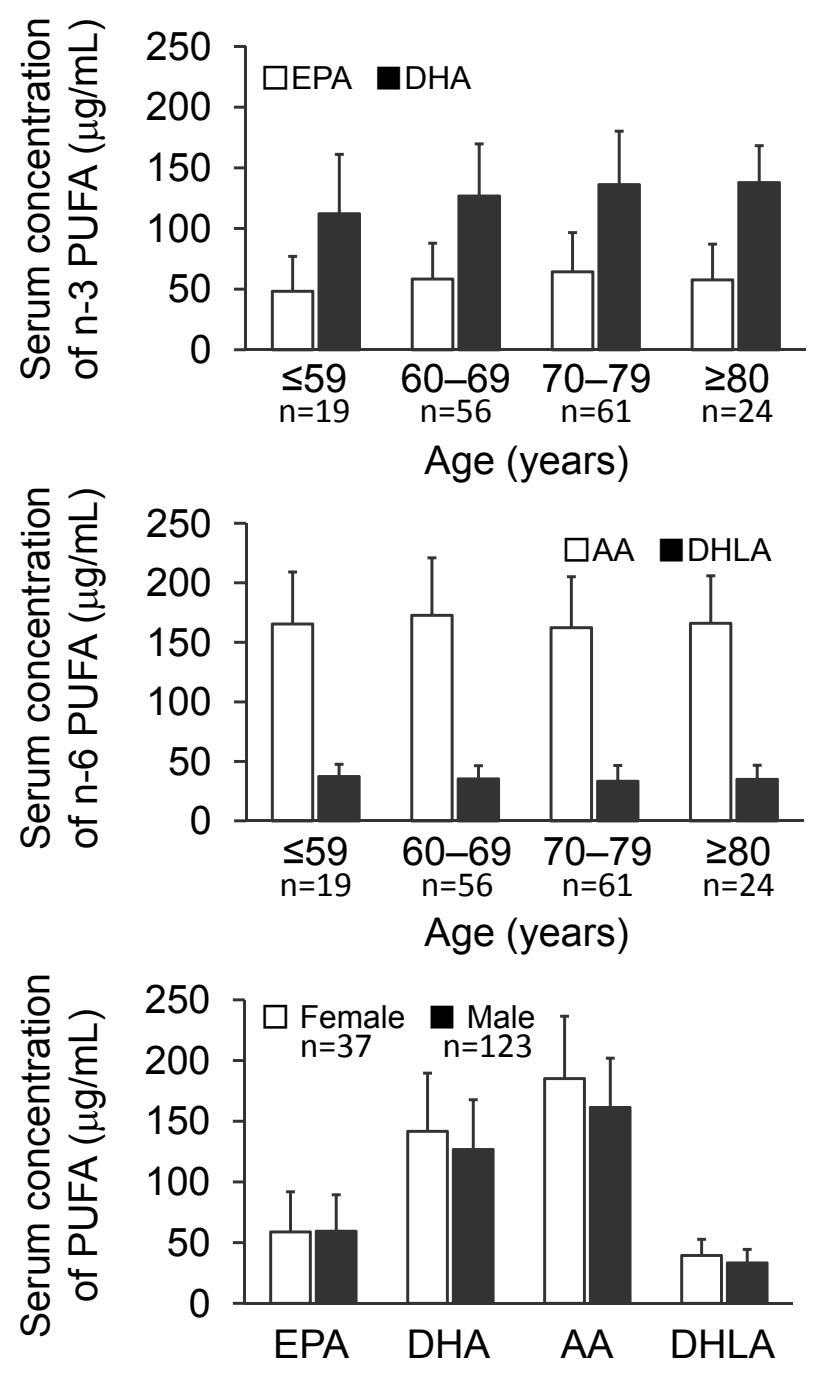

Fig. 1. Distribution of the n-3 and n-6 polyunsaturated fatty acid concentrations stratified by sex and age

The data are presented as the mean \pm SD. EPA, eicosapentaenoic acid; DHA, docosahexaenoic acid; AA, arachidonic acid; DHLA, dihomo-gamma-linolenic acid

defined as a $P$ value of $<0.05$.

\section{Results}

\section{Clinical Characteristics of the Subjects}

The patient characteristics are shown in Table 1. Of note, $83.8 \%$ of the subjects were taking HMGCoA reductase inhibitors. The distribution of the $n-3$ and n-6 PUFA concentrations stratified by sex and age is shown in Fig. 1. Although there were no significant differences, the DHA levels were higher in women than in men and the DHA levels were higher in 
Table 2. Multiple regression analysis for determinants of the percent change in the flow-mediated dilation

\begin{tabular}{lccc}
\hline Model 1 & & & \\
\hline Variable & Coefficient & $95 \%$ Confidence Interval & $P$-value \\
\hline Age & -0.08 & -0.13 to -0.04 & $<0.001$ \\
Male sex & 0.15 & -0.36 to 0.67 & 0.56 \\
Body mass index & -0.02 & -0.11 to 0.08 & 0.72 \\
Systolic blood pressure & -0.01 & -0.03 to 0.01 & 0.47 \\
Diastolic blood pressure & -0.02 & -0.06 to 0.02 & 0.41 \\
Triglycerides & -0.01 & -0.01 to 0.001 & 0.06 \\
Low-density lipoprotein cholesterol & -0.01 & -0.02 to 0.01 & 0.61 \\
High-density lipoprotein cholesterol & -0.02 & -0.05 to 0.01 & 0.08 \\
HbA1c & -0.06 & -0.46 to 0.34 & 0.77 \\
Current/past smoker & -0.13 & -0.55 to 0.29 & 0.53 \\
DHA & 0.02 & 0.01 to 0.03 & $<0.01$ \\
EPA & -0.02 & -0.03 to 0.001 & 0.06 \\
AA & 0.003 & -0.01 to 0.01 & 0.63 \\
DHLA & -0.02 & -0.06 to 0.02 & 0.31 \\
\hline
\end{tabular}

HbA1c, hemoglobin A1c; DHA, docosahexaenoic acid; EPA, eicosapentaenoic acid; AA, arachidonic acid; DHLA, dihomo-gamma-linolenic acid

Model 2

\begin{tabular}{lccc}
\hline Variable & Coefficient & 95\% Confidence Interval & $P$-value \\
\hline Age & -0.08 & -0.13 to -0.03 & $<0.001$ \\
Male sex & 0.08 & -0.42 to 0.59 & 0.74 \\
Body mass index & -0.01 & -0.11 to 0.08 & 0.80 \\
Systolic blood pressure & -0.01 & -0.03 to 0.01 & 0.49 \\
Diastolic blood pressure & -0.01 & -0.06 to 0.03 & 0.45 \\
Triglycerides & -0.01 & -0.01 to 0.001 & 0.09 \\
Low-density lipoprotein cholesterol & -0.01 & -0.02 to 0.01 & 0.62 \\
High-density lipoprotein cholesterol & -0.01 & -0.03 to 0.01 & 0.31 \\
HbA1c & -0.08 & -0.49 to 0.33 & 0.71 \\
Current/past smoker & -0.11 & -0.53 to 0.30 & 0.59 \\
DHA/AA & 1.70 & -0.10 to 3.51 & 0.06 \\
EPA/AA & -1.39 & -3.67 to 0.88 & 0.23 \\
\hline
\end{tabular}

HbA1c, hemoglobin A1c; DHA, docosahexaenoic acid; AA, arachidonic acid; EPA, eicosapentaenoic acid

patients $\geq 70$ years age than in those $<70$ years of age.

\section{Correlation between n-3 PUFA and FMD}

The single regression analysis showed no relationship between the FMD and the serum levels of fatty acids, including EPA, DHA, AA and DHLA (data not shown). A multiple regression analysis was performed to identify determinants of FMD. Consequently, age $(P<0.001)$ was found to be a negative contributor and the DHA level $(P<0.01)$ was found to be a positive contributor to the FMD. In contrast, sex, body mass index, systolic and diastolic blood pres- sure, current/past smoking and the levels of HbA1c, triglycerides, LDL-C, HDL-C, EPA, AA and DHLA did not show any statistically significant correlations (Model 1 in Table 2). Neither the EPA/AA nor DHA/ AA ratio were determinants of the FMD in the CAD patients (Model 2 in Table 2). In addition, the subanalyses of men and patients taking HMG-CoA reductase inhibitors demonstrated age to be a negative contributor and the DHA and DHA/AA levels to be positive contributors to the FMD (Supplemental Tables 1 and 2). Due to the small number of patients, subanalyses of women and patients not taking HMG- 
CoA reductase inhibitors and were not performed.

\section{Discussion}

The present study demonstrated that the serum levels of DHA contribute significantly to increased FMD values in patients with CAD. In particular, the present subanalysis of patients taking HMG-CoA reductase inhibitors showed that the DHA and DHA/ AA levels are positive contributors to an increased FMD. These results indicate that a low DHA level is a residual risk factor for endothelial dysfunction in CAD patients taking HMG-CoA reductase inhibitors.

In addition to its triglyceride-lowering effects, n-3 PUFA has several positive effects on the cardiovascular system, including effects on endothelial nitric oxide synthase (eNOS) enhancement ${ }^{16)}$ and antiinflammatory actions. It has also been reported that n-3 PUFA increases the expression and activation of eNOS, leading to vasodilation ${ }^{17)}$. Furthermore, the EPA-induced translocation of eNOS from caveolin in the cell membrane to the cytosol results in the enhancement and activation of the eNOS system and may a cause of EPA-induced vasodilation ${ }^{18)}$. Meanwhile, DHA has been reported to decrease the expression of proinflammatory cytokines (e.g. tumor necrosis factor- $\alpha$, interleukin $1 \beta$ and interleukin-6) and cell adhesion molecules (e.g. vascular cell adhesion molecule 1, E-selectin and intercellular adhesion molecule 1 ), which may have protective effects on the endothelium ${ }^{19,20)}$.

Many clinical studies have demonstrated the effects of fish oil or n-3 PUFA on the incidence of cardiovascular events; however, the differential effects of DHA and EPA are less well established ${ }^{21)}$. It has been reported that a low level of DHA is associated with all-cause death, although this relationship is not observed in patients with a low level of EPA after acute myocardial infarction ${ }^{8)}$, and that higher plasma levels of DHA, but not EPA, are associated with a reduced progression of coronary atherosclerosis in women with $\mathrm{CAD}^{22)}$. These findings suggests that DHA exerts a protective effect on CAD and indicates the possibility that the administration of DHA may reduce the onset of cardiovascular events.

Several previous studies have shown the effects of DHA on the endothelial function. For example, a double-blind placebo-controlled trial showed that DHA, but not EPA, enhances vasodilation in response to acetylcholine, which induces endogenous $\mathrm{NO}$ release $^{23,24)}$. Another study showed that DHA supplementation increases the FMD values in hyperlipidemic children ${ }^{25}$. Hence, the positive effects of $n-3$
PUFA on cardiovascular events may occur via DHAinduced improvements in the endothelial function.

In addition, DHA achieves better tissue distribution than EPA, which exhibits very little dispersion into tissue ${ }^{26,27,28)}$. The properties of being a bioavailable fatty acid may explain the preferable effects of DHA.

A large clinical trial recently provided evidence of the effects of EPA or fish oil on reducing cardiovascular events ${ }^{29)}$; however, there have been no large clinical trials showing the positive effects of DHA on cardiovascular events. Therefore, large clinical cohort studies are needed to clarify the differential effects of the components of n-3 PUFA on the endothelial function and incidence of cardiovascular events.

This study is associated with several limitations. First, this study was a retrospective study with a small sample size at a single center. Second, the cross-sectional nature of the study design does not allow for evaluations of the direct effects of DHA on the endothelial function.

\section{Conclusion}

The serum levels of DHA, but not EPA, are associated with the endothelial function evaluated according to the FMD in patients with CAD, thus suggesting that a low serum DHA level may be a predictive biomarker for endothelial dysfunction.

\section{Acknowledgements}

We thank the staff of the Hospital Information Center of Tokushima University Hospital for abstracting the patient data from the medical records. We also thank Ms. Hiromi Kawano, Ms. Hazuki Hiraoka and Ms. Mitsuyo Sato for their technical help in measuring the FMD values. This work was supported in part by JSPS KAKENHI (Grant Numbers 25461131 and 25860605).

\section{Disclosure}

The authors declare that they have no conflicts of interest to disclose.

\section{References}

1) Miller M, Cannon CP, Murphy SA, Qin J, Ray KK, Braunwald E, Prove It-Timi Investigators: Impact of triglyceride levels beyond low-density lipoprotein cholesterol after acute coronary syndrome in the PROVE IT-TIMI 22 trial. J Am Coll Cardiol, 2008; 51: 724-730 
2) Huang AL, Silver AE, Shvenke E, Schopfer DW, Jahangir E, Titas MA, Shpilman A, Menzoian JO, Watkins MT, Raffetto JD, Gibbons G, Woodson J, Shaw PM, Dhadly M, Eberhardt RT, Keaney JF, Gokce N, Vita JA: Predictive value of reactive hyperemia for cardiovascular events in patients with peripheral arterial disease undergoing vascular surgery. Arterioscler Thromb Vasc Biol, 2007; 27: 2113-2119

3) Joannides R, Haefeli WE, Linder L, Richard V, Bakkali $\mathrm{EH}$, Thuillez C, Luscher TF: Nitric-Oxide is responsible for flow-dependent dilatation of human peripheral conduit arteries in-vivo. Circulation, 1995; 91: 1314-1319

4) Gnasso A, Carallo C, Irace C, De Franceschi MS, Mattioli PL, Motti C, Cortese C: Association between wall shear stress and flow-mediated vasodilation in healthy men. Atherosclerosis, 2001; 156: 171-176

5) Lerman A, Zeiher AM: Endothelial function - Cardiac events. Circulation, 2005; 111: 363-368

6) Witte DR, Westerink J, de Koning EJ, van der Graaf Y, Grobbee DE, Bots ML: Is the association between flowmediated dilation and cardiovascular risk limited to lowrisk populations? J Am Coll Cardiol, 2005; 45: 19871993

7) Amano T, Matsubara T, Uetani T, Kato M, Kato B, Yoshida T, Harada K, Kumagai S, Kunimura A, Shinbo Y, Kitagawa $\mathrm{K}$, Ishii $\mathrm{H}$, Murohara $\mathrm{T}$ : Impact of omega-3 polyunsaturated fatty acids on coronary plaque instability: an integrated backscatter intravascular ultrasound study. Atherosclerosis, 2011; 218: 110-116

8) Hara M, Sakata Y, Nakatani D, Suna S, Usami M, Matsumoto S, Hamasaki T, Doi Y, Nishino M, Sato H, Kitamura T, Nanto S, Hori M, Komuro I, Osaka Acute Coronary Insufficiency Study (OACIS) Investigators: Low levels of serum n-3 polyunsaturated fatty acids are associated with worse heart failure-free survival in patients after acute myocardial infarction. Circ J, 2013; 77: 153-162

9) Wang QQ, Liang XH, Wang LY, Lu XF, Huang JF, Cao J, Li HF, Gu DF: Effect of omega-3 fatty acids supplementation on endothelial function: A meta-analysis of randomized controlled trials. Atherosclerosis, 2012; 221: 536543

10) Tagawa H, Shimokawa H, Tagawa T, Kuroiwa-Matsumoto M, Hirooka Y, Takeshita A: Long-term treatment with eicosapentaenoic acid augments both nitric oxidemediated and non-nitric oxide-mediated endotheliumdependent forearm vasodilatation in patients with coronary artery disease. J Cardiovasc Pharmacol, 1999; 33: 633-640

11) Deckelbaum RJ: n-6 and n-3 Fatty acids and atherosclerosis: ratios or amounts? Arterioscler Thromb Vasc Biol, 2010; 30: 2325-2326

12) Ogihara T, Kikuchi K, Matsuoka H, Fujita T, Higaki J, Horiuchi M, Imai Y, Imaizumi T, Ito S, Iwao H, Kario K, Kawano Y, Kim-Mitsuyama S, Kimura G, Matsubara H, Matsuura H, Naruse M, Saito I, Shimada K, Shimamoto K, Suzuki H, Takishita S, Tanahashi N, Tsuchihashi T, Uchiyama M, Ueda S, Ueshima H, Umemura S, Ishimitsu T, Rakugi H: The Japanese Society of Hypertension Guidelines for the Management of Hypertension (JSH 2009). Hypertens Res, 2014; 37: 599
13) Corretti MC, Anderson TJ, Benjamin EJ, Celermajer D, Charbonneau F, Creager MA, Deanfield J, Drexler H, Gerhard-Herman M, Herrington D, Vallance P, Vita J, Vogel R, International Brachial Artery Reactivity Task F: Guidelines for the ultrasound assessment of endothelialdependent flow-mediated vasodilation of the brachial artery: a report of the International Brachial Artery Reactivity Task Force. J Am Coll Cardiol, 2002; 39: 257-265

14) Maruhashi T, Soga J, Fujimura N, Idei N, Mikami S, Iwamoto Y, Kajikawa M, Matsumoto T, Hidaka T, Kihara Y, Chayama K, Noma K, Nakashima A, Goto C, Higashi Y: Nitroglycerine-induced vasodilation for assessment of vascular function: a comparison with flow-mediated vasodilation. Arterioscler Thromb Vasc Biol, 2013; 33: 14011408

15) Matsuzaki M, Yokoyama M, Saito Y, Origasa H, Ishikawa Y, Oikawa S, Sasaki J, Hishida H, Itakura H, Kita T, Kitabatake A, Nakaya N, Sakata T, Shimada K, Shirato K, Matsuzawa $Y$, Investigators J: Incremental effects of eicosapentaenoic acid on cardiovascular events in HMG-CoA reductase inhibitors -treated patients with coronary artery disease. Circ J, 2009; 73: 1283-1290

16) Dimitrow PP, Jawien M: Pleiotropic, cardioprotective effects of omega-3 polyunsaturated fatty acids. Mini Rev Med Chem, 2009; 9: 1030-1039

17) Chen J, Shearer GC, Chen Q, Healy CL, Beyer AJ, Nareddy VB, Gerdes AM, Harris WS, O'Connell TD, Wang D: Omega-3 fatty acids prevent pressure overloadinduced cardiac fibrosis through activation of cyclic GMP/protein kinase $G$ signaling in cardiac fibroblasts. Circulation, 2011; 123: 584-593

18) Omura M, Kobayashi S, Mizukami Y, Mogami K, Todoroki-Ikeda N, Miyake T, Matsuzaki M: Eicosapentaenoic acid (EPA) induces $\mathrm{Ca}(2+)$-independent activation and translocation of endothelial nitric oxide synthase and endothelium-dependent vasorelaxation. FEBS Lett, 2001; 487: 361-366

19) Weldon SM, Mullen AC, Loscher CE, Hurley LA, Roche HM: Docosahexaenoic acid induces an anti-inflammatory profile in lipopolysaccharide-stimulated human THP-1 macrophages more effectively than eicosapentaenoic acid. J Nutr Biochem, 2007; 18: 250-258

20) De Caterina R, Liao JK, Libby P: Fatty acid modulation of endothelial activation. Am J Clin Nutr, 2000; 71: 213S-223S

21) Siegel G, Ermilov E: Omega-3 fatty acids: benefits for cardio-cerebro-vascular diseases. Atherosclerosis, 2012; 225: 291-295

22) Erkkila AT, Matthan NR, Herrington DM, Lichtenstein AH: Higher plasma docosahexaenoic acid is associated with reduced progression of coronary atherosclerosis in women with CAD. J Lipid Res, 47: 2814-2819

23) Mori TA, Watts GF, Burke V, Hilme E, Puddey IB, Beilin LJ: Differential effects of eicosapentaenoic acid and docosahexaenoic acid on vascular reactivity of the forearm microcirculation in hyperlipidemic, overweight men. Circulation, 2000; 102: 1264-1269

24) Kelley DS, Adkins Y: Similarities and differences between the effects of EPA and DHA on markers of atherosclerosis in human subjects. Proc Nutr Soc, 2012; 71: 322-331 
25) Engler MM, Engler MB, Malloy M, Chiu E, Besio D, Paul S, Stuehlinger M, Morrow J, Ridker P, Rifai N, Mietus-Snyder M: Docosahexaenoic acid restores endothelial function in children with hyperlipidemia: results from the EARLY study. Int J Clin Pharmacol Ther, 2004; 42: 672-679

26) Owen AJ, Peter-Przyborowska BA, Hoy AJ, McLennan PL: Dietary fish oil dose- and time-response effects on cardiac phospholipid fatty acid composition. Lipids, 2004; 39: 955-961

27) Metcalf RG, James MJ, Gibson RA, Edwards JR, Stubberfield J, Stuklis R, Roberts-Thomson K, Young GD, Cleland LG: Effects of fish-oil supplementation on myo- cardial fatty acids in humans. Am J Clin Nutr, 2007; 85: 1222-1228

28) Arterburn LM, Hall EB, Oken H: Distribution, interconversion, and dose response of $\mathrm{n}-3$ fatty acids in humans. Am J Clin Nutr, 2006; 83: 1467S-1476S

29) Yokoyama M, Origasa H, Matsuzaki M, Matsuzawa $Y$, Saito Y, Ishikawa Y, Oikawa S, Sasaki J, Hishida H, Itakura H, Kita T, Kitabatake A, Nakaya N, Sakata T, Shimada K, Shirato K, Japan EPA lipid intervention study (JELIS) Investigators: Effects of eicosapentaenoic acid on major coronary events in hypercholesterolaemic patients (JELIS): a randomised open-label, blinded endpoint analysis. Lancet, 2007; 369: 1090-1098

Supplemental Table 1. Multiple regression analysis for determinants of the percent change in the flow-mediated dilation in the patients taking HMG-CoA reductase inhibitors

\begin{tabular}{lccc}
\hline Model 1 & & & \\
\hline Variable & Coefficient & $95 \%$ Confidence Interval & $P$-value \\
\hline Age & -0.03 & -0.04 to -0.01 & $<0.001$ \\
Male sex & 0.01 & -0.16 to 0.18 & 0.90 \\
Body mass index & -0.01 & -0.04 to 0.01 & 0.57 \\
Systolic blood pressure & -0.52 & -1.52 to 0.49 & 0.31 \\
Diastolic blood pressure & -0.01 & -0.02 to 0.01 & 0.32 \\
Triglycerides & -0.29 & -0.60 to 0.03 & 0.07 \\
Low-density lipoprotein cholesterol & -0.01 & -0.01 to 0.01 & 0.60 \\
High-density lipoprotein cholesterol & -0.23 & -0.71 to 0.26 & 0.36 \\
HbA1c & -0.30 & -1.14 to 0.53 & 0.48 \\
Current/past smoker & -0.03 & -0.17 to 0.11 & 0.66 \\
DHA & 0.64 & 0.13 to 1.14 & 0.01 \\
EPA & -0.27 & -0.56 to 0.02 & 0.07 \\
AA & 0.0003 & -0.003 to 0.004 & 0.85 \\
DHLA & -0.01 & -0.02 to 0.01 & 0.27 \\
\hline
\end{tabular}

HbA1c, hemoglobin A1c; DHA, docosahexaenoic acid; EPA, eicosapentaenoic acid; AA, arachidonic acid; DHLA, dihomo-gamma-linolenic acid

Model 2

\begin{tabular}{lccc}
\hline Variable & Coefficient & 95\% Confidence Interval & P-value \\
\hline Age & -0.02 & -0.04 to -0.01 & $<0.001$ \\
Male sex & -0.01 & -0.16 to 0.16 & 0.99 \\
Body mass index & -0.01 & -0.04 to 0.02 & 0.69 \\
Systolic blood pressure & -0.53 & -1.5 to 0.45 & 0.28 \\
Diastolic blood pressure & -0.01 & -0.02 to 0.01 & 0.36 \\
Triglycerides & -0.29 & -0.56 to -0.03 & 0.03 \\
Low-density lipoprotein cholesterol & -0.01 & -0.01 to 0.01 & 0.46 \\
High-density lipoprotein cholesterol & -0.14 & -0.59 to 0.31 & 0.53 \\
HbA1c & -0.31 & -1.15 to 0.53 & 0.46 \\
Current/past smoker & -0.04 & -0.18 to 0.10 & 0.57 \\
DHA/AA & 0.49 & 0.03 to 0.95 & 0.03 \\
EPA/AA & -0.21 & -0.49 to 0.07 & 0.14 \\
\hline
\end{tabular}

HbA1c, hemoglobin A1c; DHA, docosahexaenoic acid; AA, arachidonic acid; EPA, eicosapentaenoic aci 
Supplemental Table 2. Multiple regression analysis for determinants of the percent change in the flow-mediated dilation in men

Model 1

\begin{tabular}{lccc}
\hline Variable & Coefficient & $95 \%$ Confidence Interval & $P$-value \\
\hline Age & -0.03 & -0.05 to -0.02 & $<0.001$ \\
Body mass index & -0.01 & -0.03 to 0.03 & 0.81 \\
Systolic blood pressure & 0.10 & -0.94 to 1.14 & 0.85 \\
Diastolic blood pressure & -0.01 & -0.03 to 0.01 & 0.06 \\
Triglycerides & -0.23 & -0.55 to 0.08 & 0.14 \\
Low-density lipoprotein cholesterol & 0.01 & -0.01 to 0.01 & 0.41 \\
High-density lipoprotein cholesterol & -0.32 & -0.81 to 0.17 & 0.20 \\
HbA1c & -0.50 & -1.34 to 0.31 & 0.22 \\
Current/past smoker & -0.03 & -0.16 to 0.11 & 0.69 \\
DHA & 0.72 & 0.19 to 1.25 & $<0.01$ \\
EPA & -0.12 & -0.43 to 0.19 & 0.43 \\
AA & -0.01 & -0.01 to 0.01 & 0.37 \\
DHLA & -0.01 & -0.02 to 0.01 & 0.45 \\
\hline
\end{tabular}

HbA1c, hemoglobin A1c; DHA, docosahexaenoic acid; EPA, eicosapentaenoic acid; AA, arachidonic acid; DHLA, dihomo-gamma-linolenic acid

Model 2

\begin{tabular}{lccc}
\hline Variable & Coefficient & $95 \%$ Confidence Interval & $P$-value \\
\hline Age & -0.03 & -0.05 to -0.02 & $<0.001$ \\
Body mass index & -0.01 & -0.03 to 0.03 & 0.88 \\
Systolic blood pressure & -0.01 & -1.00 to 0.98 & 0.99 \\
Diastolic blood pressure & -0.01 & -0.02 to 0.01 & 0.08 \\
Triglycerides & -0.22 & -0.48 to 0.04 & 0.10 \\
Low-density lipoprotein cholesterol & 0.01 & -0.01 to 0.01 & 0.59 \\
High-density lipoprotein cholesterol & -0.21 & -0.64 to 0.22 & 0.33 \\
HbAlc & -0.50 & -1.31 to 0.32 & 0.23 \\
Current/past smoker & -0.03 & -0.16 to 0.10 & 0.68 \\
DHA/AA & 0.62 & 0.14 to 1.10 & 0.01 \\
EPA/AA & -0.08 & -0.37 to 0.22 & 0.60 \\
\hline
\end{tabular}

HbA1c, hemoglobin A1c; DHA, docosahexaenoic acid; AA, arachidonic acid; EPA, eicosapentaenoic acid 